Supplementary Information

\title{
Adsorption of myoglobin and corona formation on silica nanoparticles
}

Jin Gyun Lee ${ }^{1}$, Kelly Lannigan ${ }^{1,2}$, William A. Shelton ${ }^{1,3}$, Jens Meissner ${ }^{4}$, and Bhuvnesh Bharti ${ }^{1, *}$

${ }^{1}$ Cain Department of Chemical Engineering, Louisiana State University, Baton Rouge, LA

${ }^{2}$ Department of Biomedical Engineering, Bucknell University, Lewisburg, PA

${ }^{3}$ Center for Computation and Technology, Louisiana State University, Baton Rouge, LA

${ }^{4}$ Intitute for Chemistry, Technishe Universitaet Berlin, Germany

*Corresponding author: bbharti@lsu.edu 

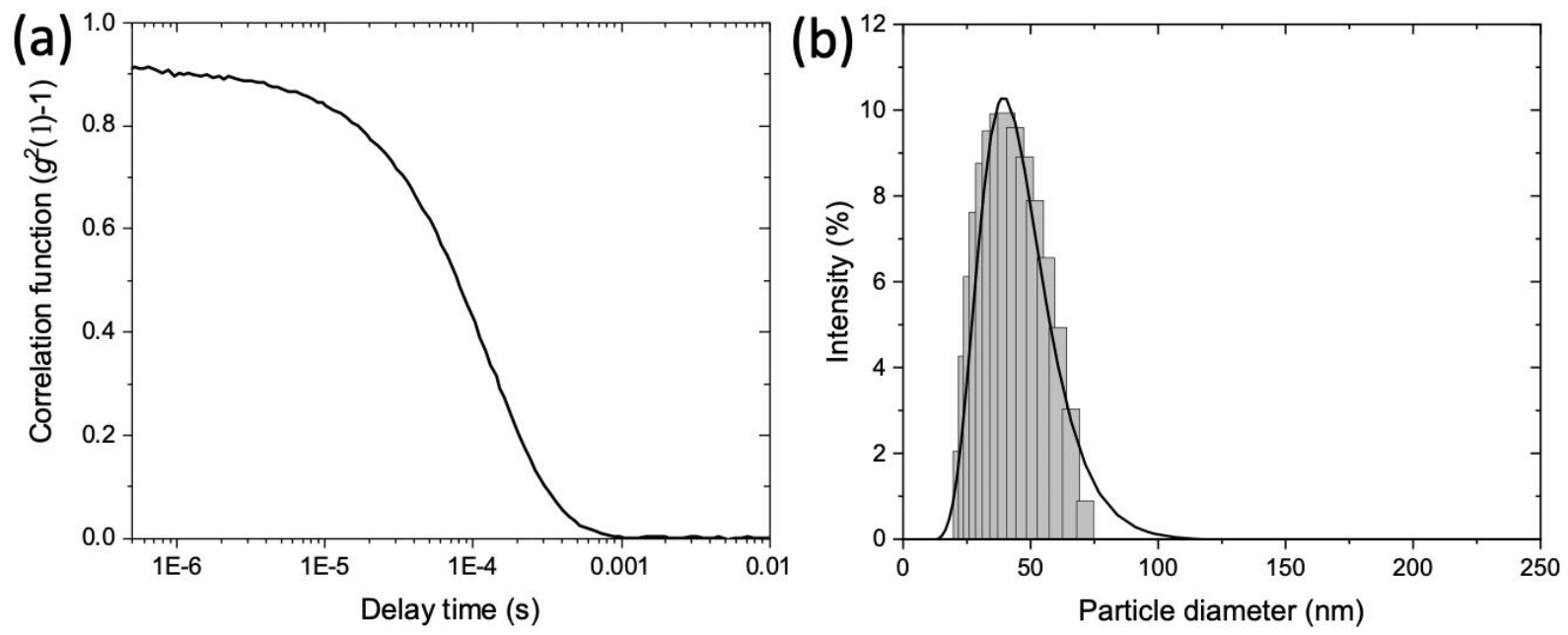

Figure S1. (a) Time-correlation function for aqueous dispersion of silica nanoparticles measured using dynamic light scattering. (b) The intensity-based size distribution of silica nanoparticles determined from the correlation function shown in (a). The size distribution shows that the nanoparticle diameter is $\sim 30 \mathrm{~nm}$ with polydispersity index of $\sim 0.1$.

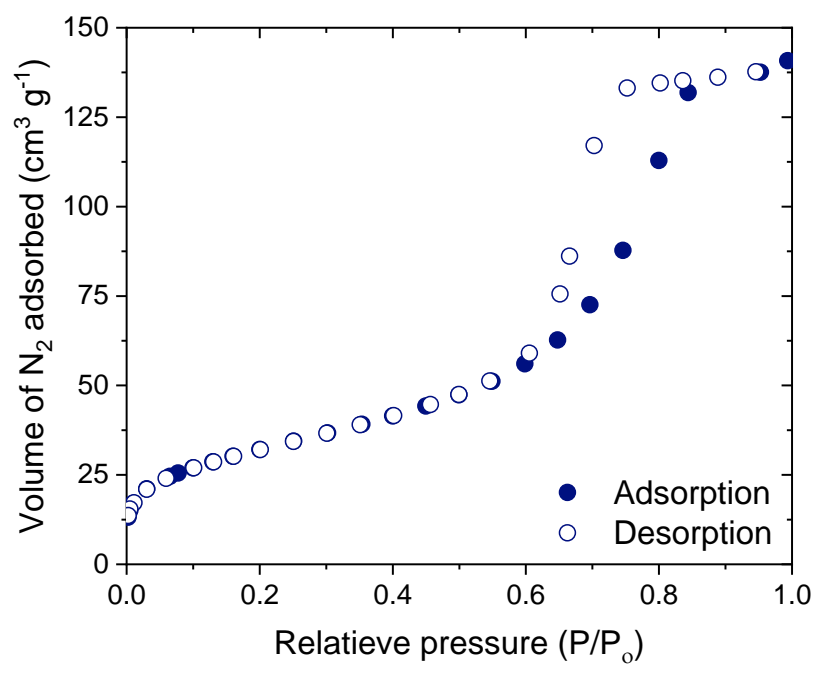

Figure S2. Nitrogen gas adsorption isotherm on dry silica nanoparticles. The specific surface area of the nanoparticles is estimated to be $114 \mathrm{~m}^{2} \mathrm{~g}^{-1}$ based on BET analysis of the nitrogen gas adsorption. 


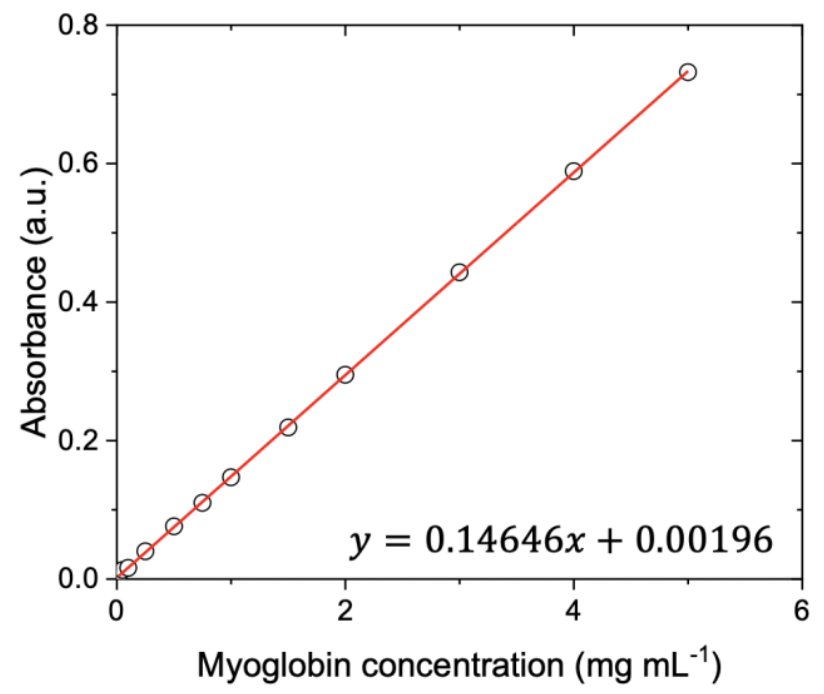

Figure S3. Calibration curve of myoglobin in water. The black circles are the experimental data, and the red line represents the best fit (given in inset) using a linear model. The straight line representing a linear increase of absorbance in a function of myoglobin concentration which is in agreement with the Beer-Lambert law. 

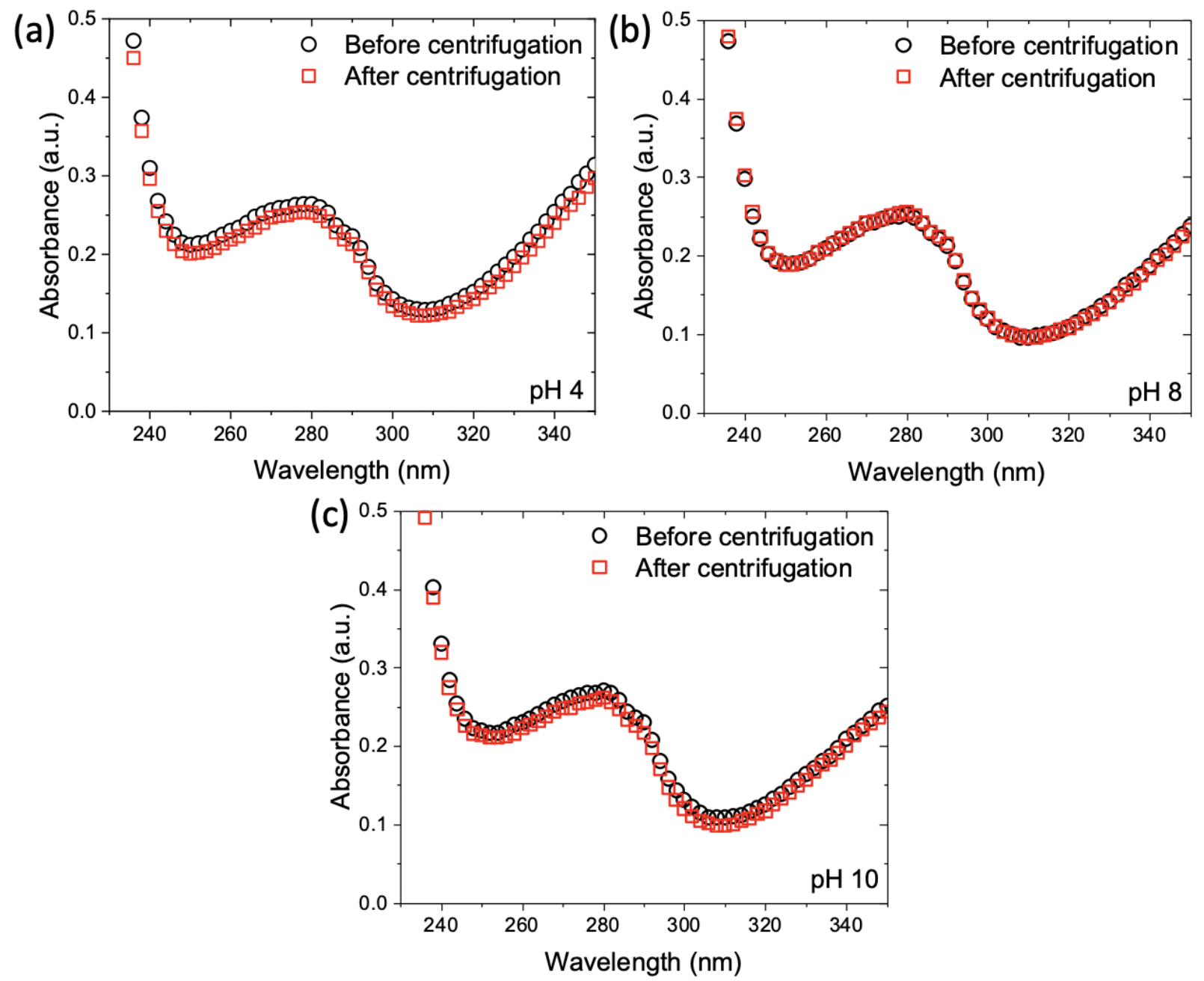

Figure S4. Absorption spectrum of myoglobin (conc. $=1.5 \mathrm{mg} \mathrm{mL}^{-1}, \mathrm{pH} 4$ (a), 8 (b), 10 (c)) before (circles) and after centrifugation at $18,100 \mathrm{~g}$ for two hours (red squares). The overlapping nature of the spectrum highlights lack of aggregation and separation of protein. 